\title{
Dust devil vortex generation from convective cells
}

\author{
O. Onishchenko ${ }^{1,2}$, O. Pokhotelov ${ }^{1}$, W. Horton ${ }^{3}$, and V. Fedun ${ }^{4}$ \\ ${ }^{1}$ Institute of Physics of the Earth, 10 B. Gruzinskaya, 123242 Moscow, Russian Federation \\ ${ }^{2}$ Space Research Institute, 84/32, Profsoyuznaya str., 117997 Moscow, Russian Federation \\ ${ }^{3}$ Institute for Fusion Studies, The University of Texas at Austin, Austin, Texas, USA \\ ${ }^{4}$ Space Systems Laboratory, Department of Automatic Control and Systems Engineering, \\ University of Sheffield, Sheffield, S1 3JD, UK
}

Correspondence to: V. Fedun (v.fedun@ sheffield.ac.uk)

Received: 30 July 2015 - Revised: 15 October 2015 - Accepted: 27 October 2015 - Published: 4 November 2015

\begin{abstract}
We have developed a hydrodynamic theory of the nonlinear stage of dust devil generation in a convectively unstable atmosphere with large-scale seed vertical vorticity. It is shown that convective motion in such an atmosphere transforms into dust devils extremely fast. The strong vortical structure of the dust devils can be formed in a few minutes or even in a fraction of a minute. The formation process strongly depends on the convective instability growth rate and horizontal vorticity.
\end{abstract}

Keywords. Meteorology and atmospheric dynamics (convective processes)

\section{Introduction}

Dust devils are upward spiralling vortices. They are common atmospheric phenomena on Earth and Mars and, due to the presence of dust, these vortices can be visible as miniature or small tornadoes. This phenomenon can have a major impact on weather and climate and serve as nuclei of dust storms on Mars.

The main characteristics of dust devils have been analysed and collected in a number of in situ measurements (Balme and Greeley, 2006; Leovy, 2003; Ryan and Carroll, 1970; Sinclair, 1969; Thomas and Gierasch, 1985), as well as laboratory (Greeley et al., 2003; Zheng et al., 2003) and numerical experiments (e.g. Gu et al., 2008; Huang et al., 2008). It is well known that this phenomenon arises on sunny days when the superadiabatic lapse rate occurs. The latter leads to convective instability in the planetary boundary layer. This results in generation of convective cells with finite poloidal (horizontal) vorticity (e.g. Onishchenko and Pokhotelov, 2012; Onishchenko et al., 2013, 2014a, b).

Numerous observations and laboratory experiments have revealed that dust devils can be generated only in a convectively unstable atmosphere with large-scale seed vertical vorticity. Sinclair (e.g. Sinclair, 1969) has analysed dust devil generation in the lee of small mountains. Based on the analysis of observational results, he concluded that dust devils probably arise due to a vortex embedding in the background flow together with superadiabatic lapse rate.

In such structures the dust grains are in contact with each other and, therefore, via friction or triboelectric processes can generate electric charges and related electric fields (Farrell et al., 2004, 2006; Renno and Kok, 2008). The electric forces near and within dust devils are coherent and fairly strong, but nevertheless they are, as a rule, still small in comparison with corresponding pressure gradients.

The linear theory of dust devil generation, i.e. by assuming that the excited small-scale vertical vorticity is smaller than external vorticity, has been developed in Onishchenko et al. (2014c, 2015). One of the main features of dust devil vortex generation refers to a strong increase in the tangential speed and vertical vorticity within a few seconds or even a fraction of a second. In this paper we study the nonlinear stage of dust devil generation. We show that the vertical vorticity and the toroidal speed in an unstably stratified atmosphere with large-scale seed vorticity rapidly grows within a time of the order of the inverse convective instability growth rate. Note that, in the present analysis, the dissipative processes (i.e. viscosity, thermal conductivity, heat flow, etc.) are neglected. Moreover, we disregard the effects of the dust component assuming that it plays a passive role in vortex generation. 


\section{The model}

We consider the fluid motion in cylindrical geometry $(r, \phi, z)$ and the axially symmetric case $\partial / \partial \phi=0$. The most general divergence-free flow velocity $\boldsymbol{v}$ can be decomposed into its poloidal $\boldsymbol{v}_{\perp}=\left(v_{r}, 0, v_{z}\right)$ and toroidal $v_{\phi} \hat{\boldsymbol{e}}_{\phi}$ parts, $\boldsymbol{v}=$ $\boldsymbol{v}_{\perp}+v_{\phi} \hat{\boldsymbol{e}}_{\phi}$, respectively. Here $\boldsymbol{v}_{\perp}=\nabla \times(\psi \times \nabla \phi)=(\nabla \psi \times$ $\nabla \phi) / r$, where $\psi(t, r, z)$ is the stream function:

$v_{r}=-\frac{1}{r} \frac{\partial \psi}{\partial z}, \quad v_{z}=\frac{1}{r} \frac{\partial \psi}{\partial r}$.

According to Onishchenko et al. (2014c, 2015) the equation describing the nonlinear internal gravity waves in the approximation $\partial / \partial r \gg \partial / \partial z$ can be written as

$$
\left(\frac{\partial^{2}}{\partial t^{2}}+\omega_{g}^{2}\right) \Delta_{r}^{*} \psi+\frac{1}{r} \frac{\partial}{\partial t} J\left(\psi, \Delta_{r}^{*} \psi\right)=0
$$

where $\omega_{g}$ is the Brunt-Väisälä or buoyancy frequency given by

$\omega_{g}^{2}=g\left(\frac{\gamma_{a}-1}{\gamma_{a} H}+\frac{1}{T} \frac{\mathrm{d} T}{\mathrm{~d} z}\right)$,

where $J(A, B)=(\partial A / \partial r) \partial B / \partial z-(\partial A / \partial z) \partial B / \partial r$ is the Jacobian, $\gamma_{a}$ is the ratio of specific heats, $H=g / c_{s}^{2}$ is the reduced height of atmosphere, $c_{s}$ is the adiabatic sound velocity and

$\Delta_{r}^{*}=r \frac{\partial}{\partial r} \frac{1}{r} \frac{\partial}{\partial r}$.

If $\omega_{g}^{2}<0$, Eq. (2) describes the dynamics of convective cells in an unstable atmosphere. An equation similar to Eq. (2) has been obtained previously by Stenflo (1990) for interpretation of behaviour of acoustic gravity vortices.

In this work we constrain the model stream function as

$\psi=\frac{\alpha r^{2} z}{2} \exp \left(\gamma t-r^{2} / r_{0}^{2}\right)$,

where $r_{0}$ is the vortex radius, $\gamma$ is the growth rate of the rising air, and $\alpha$ is a constant. By applying the differential operator Eq. (4) to the stream function Eq. (5) it is easy to obtain that

$\Delta_{r}^{*} \psi=-\frac{8}{r_{0}^{2}} \psi+\frac{4}{r_{0}^{2}} \frac{r^{2}}{r_{0}^{2}} \psi$

Therefore, by taking into account Eq. (6) the nonlinear term in Eq. (2) is negligible in comparison with the linear terms if

$\frac{\alpha}{\gamma} \frac{r^{2}}{r_{0}^{2}} \exp \left(\gamma t-r^{2} / r_{0}^{2}\right) \ll 1$

In this approximation from Eq. (2), it follows that $\gamma=\left|\omega_{g}\right|$. Thus, the function in Eq. (5) is a solution of Eq. (2) if the condition in Eq. (7) is satisfied.
Using Eq. (5) the radial and vertical velocities can be written as

$v_{r}=-\frac{\alpha r}{2} \exp \left(\gamma t-r^{2} / r_{0}^{2}\right)$

and

$v_{z}=\alpha z\left(1-\frac{r^{2}}{r_{0}^{2}}\right) \exp \left(\gamma t-r^{2} / r_{0}^{2}\right)$

From Eqs. (8) and (9), it follows that the poloidal vorticity at $r \ll r_{0}$ is

$\omega_{\phi}=\frac{\partial v_{r}}{\partial z}-\frac{\partial v_{z}}{\partial r}=4 \alpha \frac{r z}{r_{0}^{2}} \exp (\gamma t)$.

Thus, the parameter $\alpha$ corresponds to horizontal (poloidal) vorticity for $4 r z=r_{0}^{2}$ and $t=0$ in the internal vortex region. To investigate the evolution of the vertical (toroidal) vorticity $\omega_{z}$ we make use of the following equation:

$\frac{\partial \omega_{z}}{\partial t}+v_{r} \frac{\partial \omega_{z}}{\partial r}+v_{r} \frac{\partial \omega_{z}}{\partial z}=\omega_{z} \frac{\partial v_{z}}{\partial z}$,

which describes the interaction of vertical vorticity with poloidal motion. We consider that in the vortex area there is a stationary large scale, with characteristic scale $R \gg r_{0}$, vortex vorticity germ $\Omega_{z h}(r)$ when $z<h$ and $\Omega_{z}(r)$ when $z>h$. Here $h$ is the vertical scale due to the interaction of vortex with the ground surface,

$\Omega_{z h}(r)=\Omega \frac{z}{h}\left[1-\exp \left(\frac{r^{2}}{R^{2}}\right)\right] \simeq \Omega \frac{z}{h} \frac{r^{2}}{R^{2}}$,

and

$\Omega_{z}(r)=\Omega\left[1-\exp \left(\frac{r^{2}}{R^{2}}\right)\right] \simeq \Omega \frac{r^{2}}{R^{2}}$,

where $\Omega$ is a constant. We assume that $\omega_{z}=\Omega_{z h} f(r, t)$ and $\omega_{z}=\Omega_{z} F(r, t)$ when $z<h$ or $z>h$, respectively. By substituting expressions for $\omega_{z}$ into Eq. (11) one can obtain that

$f(r, t)=\exp \left[(\alpha / \gamma) \exp \left(\gamma t-r^{2} / r_{0}^{2}\right)\right]$

and

$F(r, t)=\exp \left[2(\alpha / \gamma) \exp \left(\gamma t-r^{2} / r_{0}^{2}\right)\right]$.

It is seen that in the internal vortex region at $r^{2} / r_{0}^{2} \ll 1$ and when $t=0$, we have $f(r, t)=F(r, t)=1$. The vortex generation when $F(r, t)-1<1$ and therefore $F(r, t)=$ $1+2(\alpha / \gamma) \exp \left(\gamma t-r^{2} / r_{0}^{2}\right)$ corresponds to the linear approximation which has been investigated before by Onishchenko et al. (2014c, 2015). In this article we study the vertical vortex generation at arbitrary values $f(r, t)$ and $F(r, t)$ and pay special attention to the nonlinear stage of the vertical vorticity $\omega_{z}$ generation when in the internal vortex region 

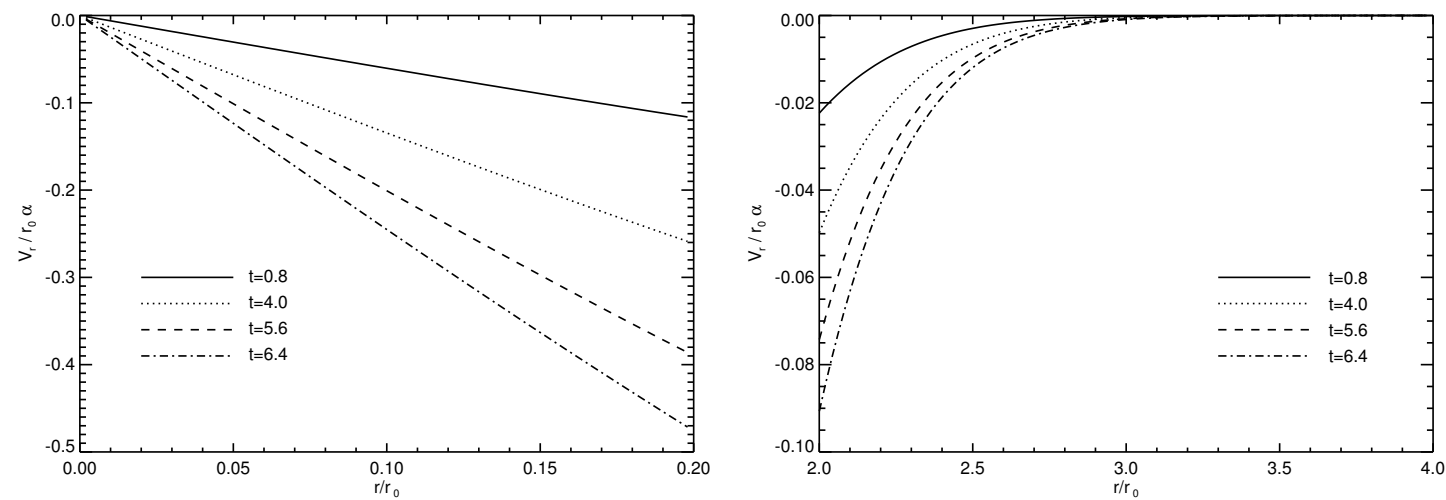

Figure 1. The normalized radial velocity $\left(V_{r} / r_{0} \alpha\right)$ as a function of $r / r_{0}$ and time $t=0.8,4.0,5.6$, and $6.4 \mathrm{~s}$. The $r / r_{0}$ dependance is shown for two separate radial intervals: $0<r / r_{0}<0.2$ (left) and $2<r / r_{0}<4$ (right).
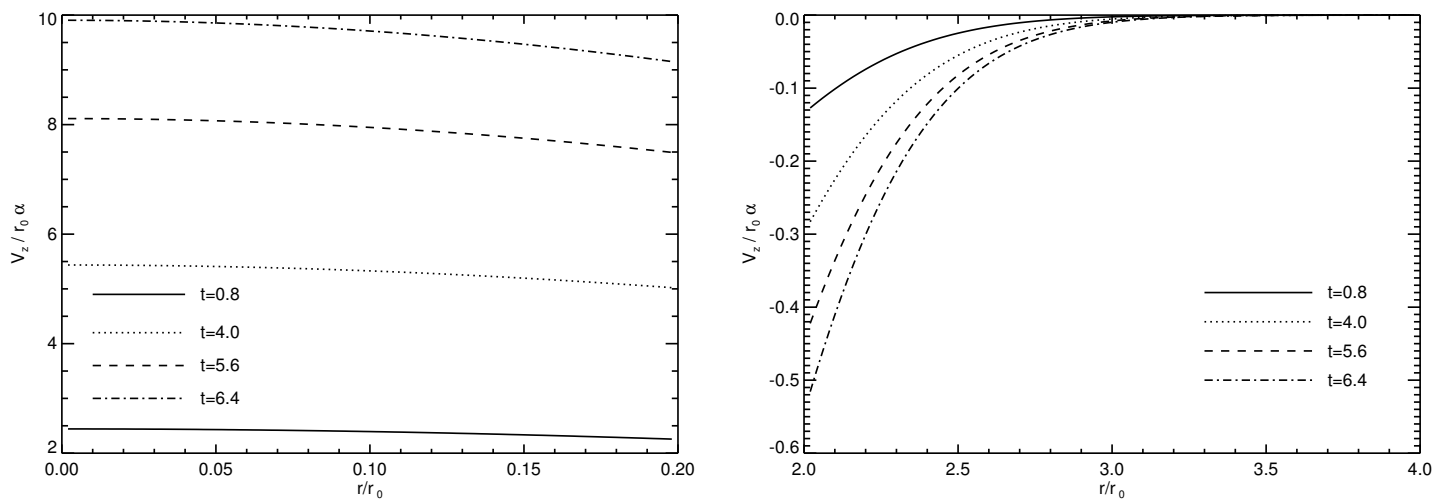

Figure 2. The normalized vertical velocity $\left(V_{z} / r_{0} \alpha\right)$ for $z=2 r_{0}$ as a function of $r / r_{0}$ and time $t=0.8,4.0,5.6$, and 6.4 s. The $r / r_{0}$ intervals are the same as for Fig. 1.

$f(r, t) \gg 1$ and $F(r, t) \gg 1$. The vertical vorticity $\omega_{z}$ is related to tangential velocity $v_{\phi}$ by relation

$\omega_{z}=\frac{1}{r}\left(\frac{\partial}{\partial r} r v_{\phi}\right)$.

Thus the tangential velocity for $z<h$ is as follows:

$v_{\phi}=v_{\phi h 0} \exp \left[(\alpha / \gamma) \exp \left(\gamma t-r^{2} / r_{0}^{2}\right)\right]$.

For $z>h$,

$v_{\phi}=v_{\phi 0} \exp \left[2(\alpha / \gamma) \exp \left(\gamma t-r^{2} / r_{0}^{2}\right)\right]$.

Here,

$v_{\phi h 0}=(\Omega R / 4)(r / R)^{3}(z / h)$

and

$v_{\phi 0}=(\Omega R / 4)(r / R)^{3}$

are seeds of tangential velocities. To illustrate our results we make use of observations from Oke et al. (2007). We consider an air temperature lapse rate of $2 \mathrm{C} \mathrm{m}^{-1}$ that corresponds to characteristic temperature vertical scale $L_{T}=150 \mathrm{~m}$ and the growth rate $\gamma=0.25 \mathrm{~s}^{-1}$. Figures 1 and 2 show the dependence of normalized radial and vertical components of the velocity within the time interval $t \leq 4 \mathrm{~s}$ and when $\alpha=\gamma$ in the radial domains $r / r_{0} \leq 0.2$ or $r / r_{0} \geq 2.0$. Note that according to Eq. (9) the vertical velocity is proportional to $z$. Figure 2 is plotted for $z=2 r_{0}$. Equations (8) and (10) show that the poloidal (horizontal) vorticity is proportional to the radial velocity $v_{r}(r, t)$; therefore its dependence on the radial coordinate, and time is similar to that in $v_{r}(r, t)$. In the intermediate domain $0.2<r / r_{0}<2.0$, where the condition in Eq. (7) fails, the stream function in Eq. (5) is not a solution of Eq. (2). In this case while solving Eq. (2), the nonlinearity associated with the Jacobian should be taken into account. Further investigation of this nonlinearity in the region $0.2<r / r_{0}<2.0$ will be carried out elsewhere. Comparing the left and right panels of Figs. 1 and 2, using interpolation behaviour of velocities in the regions $0.2<r / r_{0}<2.0$ and $r / r_{0} \geq 2.0$, one can assume that for $r \simeq r_{0}$ the absolute values of the radial velocity and the horizontal vorticity attain their maximum values. Then the vertical component of the velocity, for $r \approx r_{0}$, vanishes and then changes the sign. 

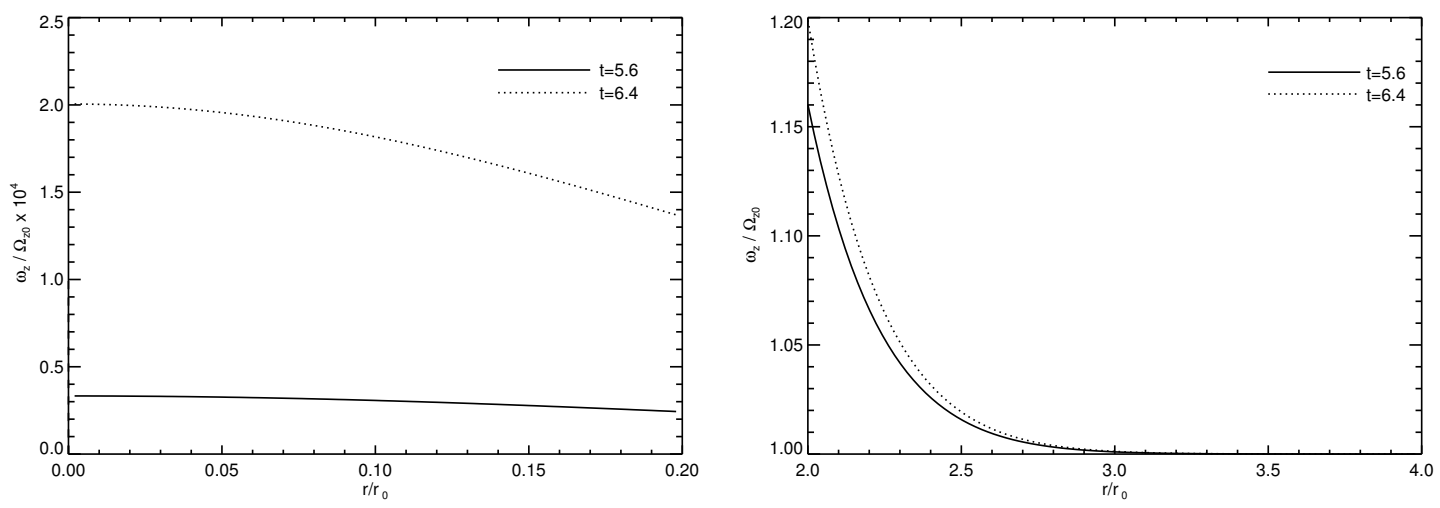

Figure 3. The radial dependence of the vertical vorticity amplification factor $\exp \left[2(\alpha / \gamma) \exp \left(\gamma t-r^{2} / r_{0}^{2}\right)\right]$ in the inner (left) and the outer region (right) of the vortex.

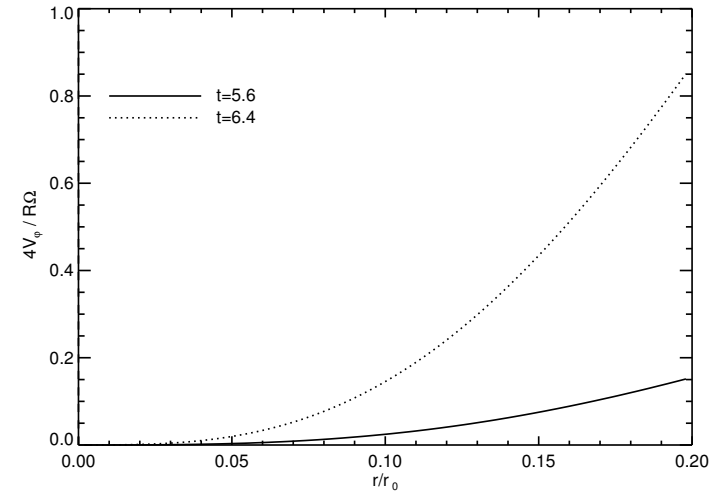

Figure 4. The normalized toroidal velocity as a function of $r / r_{0}$ in the inner vortex region for $R=50 \mathrm{~m}$ and $\Omega=0.1 \mathrm{~s}^{-1}$.

Figure 3 shows the amplification factor $F$ in the radial intervals the same as in Figs. 1 and 2. Note the strong, i.e. 10-times, increase of $F$ from $t=5.6$ to $6.4 \mathrm{~s}$ in the interior of the dust devil vortex, while in the external region (right panel) the amplification factor $F$ remains close to the unity. Therefore, the amplification of the vertical vorticity is concentrated in the internal part of the vortex. Figure 4 indicates the normalized toroidal velocity in the internal part. The example shows a factor-of- 8 increase in the toroidal velocity at $r=r_{0} / 5$ in the time interval $\delta t=6.4-5.6=0.8 \mathrm{~s}$.

For smaller temperature gradient with scale $L_{T}=300 \mathrm{~m}$ we have $\gamma=0.18 \mathrm{~s}^{-1}$, and the behaviour of $v_{r}$ and $v_{z}$ remains the same. However, the former time interval $t \leq 4 \mathrm{~s}$ is enlarged to $t \leq 9 \mathrm{~s}$ following the condition $\gamma t=$ const.

In Figs. 1-4 we show the generation of dust devils in the Earth atmosphere with its characteristic parameters. When applied to the Martian atmosphere, these parameters will have other values; as a result, the instability growth rate and spatial scales of generated dust devils will be different. Nevertheless, the behaviour of radial velocity $\left(V_{r}\right)$, vertical velocity $\left(V_{z}\right)$, vertical vorticity $\left(\omega_{z}\right)$, and toroidal velocity $\left(V_{\phi}\right)$ will remain same. Therefore, Figs. 1-4 can also be used to illustrate the mechanism of dust devil generation on Mars.

\section{Conclusion}

In summary, the new nonlinear analysis developed here shows how an unstably stratified atmosphere develops highspeed toroidal rotation from a large-scale seed vertical vorticity. It is shown that convective cells in such a case can convert into dust devil vortex structures extremely fast, more rapidly than simply exponentially. From formulae (15) and (18), it follows that the vertical vorticity $\omega_{z} / \Omega_{z 0}$ and toroidal velocity $v_{\phi} / v_{\phi 0}$ grow as $\sim \exp \left[2(\alpha / \gamma) \exp \left(\gamma t-r^{2} / r_{0}^{2}\right)\right]$. It is seen that, in the inner vortex region, $r / r_{0} \ll 1$, for moderate time domain $t \gtrsim \gamma^{-1}$ the amplification factor shows explosive behaviour if $\alpha / \gamma \gtrsim 1$.

Acknowledgements. This research is partially supported by the Program of the Russian Academy of Sciences No. 9, and by RFBR through grants 14-05-00850 and 15-05-07623. W. Horton is supported by NSF grant no. 0964692 at the University of Texas at Austin and the University of Aix-Marseille/CNRS. V. Fedun acknowledges the financial support received from the Science and Technology Facilities Council (STFC), UK.

The topical editor V. Kotroni thanks the three anonymous referees for help in evaluating this paper.

\section{References}

Balme, M. and Greeley, R.: Dust devils on Earth and Mars, Rev. Geophys., 44, RG3003, doi:10.1029/2005RG000188, 2006.

Farrell, W. M., Smith, P. H., Delory, G. T., Hillard, G. B., Marshall, J. R., Catling, D., Hecht, M., Tratt, D. M., Renno, N., Desch, M. D., Cummer, S. A., Houser, J. G., and Johnson, B.: Electric and magnetic signatures of dust devils from the 20002001 MATADOR desert tests, J. Geophys. Res., 109, E03004, doi:10.1029/2003JE002088, 2004. 
Farrell, W. M., Renno, N., Delory, G. T., Cummer, S. A., and Marshall, J. R.: Integration of electrostatic and fluid dynamics within a dust devil, J. Geophys. Res., 111, E01006, doi:10.1029/2005JE002527, 2006.

Greeley, R., Balme, M. R., Iversen, J. D., Metzger, S., Mickelson, R., Phoreman, J., and White, B.: Martian dust devils: Laboratory simulations of particle threshold, J. Geophys. Res.-Planets, 108, 5041, doi:10.1029/2002JE001987, 2003.

Gu, Z. L., Qiu, J., Zhao, Y. Z., and Li, Y.: Simulation of terrestrial dust devil patterns, Adv. Atmos. Sci., 25, 31-42 doi:10.1007/s00376-008-0031-7, 2008.

Huang, N., Yue, G., and Zheng, X.: Numerical simulations of a dust devil and the electric field in it, J. Geophys. Res., 113, D20203, doi:10.1029/2008JD010182, 2008.

Leovy, C..: Mars The devil is in the dust, Nature, 424, 6952, 10081009, 2003.

Oke, A. M. C., Tapper, N. J., and Dunkerley, D.: Willy willies in the Australian landscape: The role of key meteorological variables and surface conditions in defining frequency and spatial characteristics, J. Arid Environ., 71, 201-215, 2007.

Onishchenko, O. and Pokhotelov, O.: Generation of zonal structures by internal gravity waves in the Earth's atmosphere, Dokl. Earth Sci., 445, 845-848, doi:10.1134/S1028334X12070070, 2012.

Onishchenko, O., Pokhotelov, O., and Fedun, V.: Convective cells of internal gravity waves in the earth's atmosphere with finite temperature gradient, Ann. Geophys., 31, 459-462, doi:10.5194/angeo-31-459-2013, 2013.

Onishchenko, O. G., Pokhotelov, O. A. and Fedun, V.: Convection cells of internal gravity waves in the terrestrial atmosphere, Dokl. Earth Sci., 454, 37-39, doi:10.1134/S1028334X14010036, 2014a.
Onishchenko, O., Pokhotelov, O., Horton, W., Smolyakov, A., Kaladze, T., and Fedun, V.: Rolls of the internal gravity waves in the Earth's atmosphere, Ann. Geophys., 32, 181-186, doi:10.5194/angeo-32-181-2014, 2014b.

Onishchenko, O., Horton, W., Pokhotelov, O., and Stenflo, L.: Dust devil generation, Phys. Scripta, 89, 075606, doi:10.1088/00318949/89/7/075606, 2014c.

Onishchenko, O. G., Pokhotelov, O. A., and Horton, W.: Dust devil dynamics in the internal vortex region, Phys. Scripta, 90, 068004, doi:10.1088/0031-8949/90/6/068004, 2015.

Renno, N. O. and Kok, J. F.: Electrical Activity and Dust Lifting on Earth, Mars, and Beyond, Space Sci. Rev., 137, 419-434, doi:10.1007/s11214-008-9377-5, 2008.

Ryan, J. A. and Carroll, J. J.: Dust devil wind velocities: Mature state, J. Geophys. Res., 75, 531-541, doi:10.1029/JC075i003p00531, 1970.

Sinclair, P. C.: General Characteristics of Dust Devils, J. Appl. Meteorol., 8, 32-45, doi:10.1175/1520 0450(1969)008<0032:GCODD>2.0.CO;2, 1969.

Stenflo, L.: Acoustic gravity vortices, Phys. Scripta, 41, 641-642, doi:10.1088/0031-8949/41/5/001, 1990.

Thomas, P. C. and Gierasch, P. J.: Dust devils on Mars, Science, 230, 175-177, doi:10.1126/science.230.4722.175, 1985.

Zheng, X. J., Huang, N., and Zhou, Y.: Laboratory measurement of electrification of wind-blown sands and simulation of its effect on sand saltation movement, J. Geophys. Res.-Atmos., 108, 4322-4331, doi:10.1029/2002JD002572, 2003. 